ARTIGO ORIGINAL ORIGINAL ARTICLE

\title{
Custos do tratamento do hiperparatireoidismo secundário à doença renal crônica, com cinacalcete ou paratireoidectomia, para pacientes não controlados com a terapia clínica convencional sob a perspectiva do Sistema Único de Saúde
}

\author{
Secondary hyperparathyroidism treatment costs with cinacalcet \\ or PTX, for uncontrolled patients with conventional clinical \\ therapy under Brazilian Public Health System perspective
}

Denizar Vianna Araújo', Laura Murta Amaral², Ana Claudia Guersoni³, Aluízio Barbosa de Carvalho ${ }^{4}$, Cristina Kahrol ${ }^{5}$, Fabio Montenegro ${ }^{6}$, Leandro Lucca7 ${ }^{7}$ Elisa de Albuquerque Sampaio ${ }^{8}$, Melanie Custódio ${ }^{6}$, Rosa Maria Afonso Moyses ${ }^{9}$, Vanda Jorgetti ${ }^{6}$

DOI: 10.21115/JBES.v9.n1.p54-61

\section{Palavras-chave:}

cinacalcete, hiperparatireoidismo secundário, insuficiência renal crônica, paratireoidectomia

\section{RESUMO}

Objetivos: Estimar a população elegível para o tratamento de pacientes com hiperparatireoidismo secundário (HPTS), não controlados com terapia convencional, bem como avaliar a utilização de recursos para o tratamento dessa população com cinacalcete ou paratireoidectomia (PTX). Métodos: Utilização da técnica Delphi por painel de especialistas. A pesquisa foi realizada utilizando questionário estruturado, enviado por meio eletrônico aos especialistas, e seguida de encontro presencial. Os custos foram obtidos de bases de dados governamentais. Apenas custos médicos diretos foram incluídos, sob a perspectiva do Sistema Único de Saúde (SUS) (em reais no ano de 2014). Os dados foram avaliados pelo Microsoft Excel versão 2013. Resultados: A população no cenário de mundo real indicada para o tratamento com cinacalcete foi de 7.705 pacientes. Já a população real encaminhada para a PTX foi de 7.691 pacientes, sendo esse número 76,3\% maior que a população ideal com indicação de PTX, que foi de 1.822 pacientes. O custo estimado do tratamento com cinacalcete foi de R\$27.712,95 (considerando a dose recomendada em bula para cinacalcete, de 30 a $180 \mathrm{mg}$ / dia) e de R\$ 16.841,85 para PTX (incluindo os períodos pré e pós-cirúrgico). A análise de sensibilidade foi baseada na dose média de cinacalcete, conforme o estudo EVOLVE (66,8 mg/dia). Nesse cenário, o custo do tratamento com cinacalcete foi de R\$11.924,13 (57\% menor que o cenário com a dose de bula). Conclusão: No cenário SUS, o número de pacientes encaminhados para PTX foi 76,3\% maior que os idealmente indicados à cirurgia, o que ocorre devido à falta de opções terapêuticas.

\footnotetext{
Recebido em: 14/11/2016. Aprovado para publicação em: 27/04/2017.

1. Universidade do Estado do Rio de Janeiro (UERJ), Rio de Janeiro, RJ, Brasil.

2. Programa de Pós-Graduação em Ciências Médicas da UERJ, Rio de Janeiro, RJ, Brasil.

3. Programa de Pós-Graduação em Medicina Preventiva da Universidade de São Paulo (USP), São Paulo, SP, Brasil. Amgen Brasil,

São Paulo, SP, Brasil.

4. Universidade Federal de São Paulo (Unifesp), São Paulo, SP, Brasil.

5. Hospital das Clínicas de Porto Alegre da Universidade Federal do Rio Grande do Sul (UFRGS), Porto Alegre, RS, Brasil. 6. USP, São Paulo, SP, Brasil.

7. Hospital das Clínicas da Faculdade de Medicina de Ribeirão Preto da Universidade de São Paulo (HCFMRP-USP), Ribeirão Preto, SP, Brasil.

8. Universidade Federal Fluminense (UFF), Niterói, RJ, Brasil

9. Programa de Mestrado em Medicina da Universidade Nove de Julho (Uninove), São Paulo, SP, Brasil.

Instituição onde o trabalho foi executado: Universidade do Estado do Rio de Janeiro (UERJ).

Conflitos de interesse: Estudo realizado com financiamento da Amgem.

Autor correspondente: Denizar Vianna Araújo. Boulevard 28 de Setembro, 77, sala 329, Departamento de Clínica Médica -

Vila Isabel, Rio de Janeiro, RJ, Brasil. Telefone: +55 (21) 2868-8484. E-mail: denizar@uerj.br
} 


\section{Keywords:}

cinacalcet, secondary hyperparathyroidism, chronic kidney disease, resource use

\section{ABSTRACT}

Objectives: To estimate patient management patterns, associated medical resource utilization and use of cinacalcet for secondary hyperparathyroidism in chronic hemodialysis patients and much uncontrolled with conventional treatment, in the Unified Healthcare System (SUS) setting, in 2014. Methods: An expert panel was carried using the Delphi technique. The research was done by structured and unambiguous questionnaires that were sent by email to the entire Delphi panel, followed by a face meeting. Expense inputs were mainly obtained from government fee schedules and pharmaceutical price tables. Only medical direct costs were included under the perspective of SUS [in 2014 Brazilian Real (BRL)]. Data were analyzed using Microsoft Excel Worksheet version 2013. Results: The eligible population to cinacalcet treatment was 9,513 patients. Considering an ideal scenario, this number goes to 7,705 patients. The estimated population for parathyroidectomy was 7,691 patients in a real scenario and 1,822 in an ideal scenario (76.3\% more patients than the ideally suited to the procedure). The estimated annual cost with cinacalcet treatment is $27,712.95 \mathrm{BRL}$ (considering the label dose for cinacalcet) and 16,841.85 BRL for parathyroidectomy (including pre and post-operative period), respectively. A sensitivity analysis was performed considering the cost of cinacalcet treatment using the drug's dose of EVOLVE study (66.8 mg). This scenario showed a total cost of 11,924.13 BRL (57\% less than label dose scenario). Conclusion: $76.3 \%$ more patients are indicated to the surgery due the absence of other therapeutic options for management of secondary hyperparathyroidism in chronic hemodialysis patients and much uncontrolled with conventional treatment, in the SUS setting.

\section{Introdução}

O hiperparatireoidismo secundário (HPTS) é uma complicação frequentemente observada nos pacientes com doença renal crônica (DRC). Com a progressão da doença renal, observam-se mudanças no metabolismo mineral como hipocalcemia, hiperfosfatemia, aumento dos níveis séricos do fator de crescimento de fibroblastos-23 (FGF-23), diminuição dos níveis de 1,25-hidroxivitamina D (calcitriol) e elevação do paratormônio (PTH), constituindo o HPTS (Custódio et al., 2013; Oliveira et al., 2011). Com base no censo da Sociedade Brasileira de Nefrologia (SBN) de 2013, estima-se que, dos 100.000 pacientes em diálise no Brasil, 13\% apresentam HPTS moderado, 9\% grave e 4\% muito grave (Custódio, 2014; Cunningham et al., 2011; Custódio et al., 2013).

O diagnóstico do HPTS na prática clínica se faz pela dosagem do PTH; nos pacientes em diálise, níveis séricos superiores a $300 \mathrm{pg} / \mathrm{mL}$ são indicativos da doença. Dosagens de cálcio (Ca), fósforo (P) e fosfatase alcalina ( $F A$ ) também são consideradas para diagnóstico da gravidade da doença e como parâmetros para seguimento dos pacientes (Custódio et al., 2013).

O tratamento de HPTS está centrado no controle dos níveis de Ca, P e PTH. Segundo as Diretrizes Terapêuticas da SBN, o tratamento não farmacológico inclui dieta restrita em P e adequação da diálise, enquanto o tratamento farmacológico inclui o uso de quelantes de P (sais de Ca e/ou cloridrato de sevelâmer), de calcitriol e seus análogos, como o paricalcitol, e de calcimiméticos, como o cinacalcete (Custódio et al., 2013; Lima et al., 2011; Oliveira et al., 2011).

Os pacientes que não respondem ao tratamento clínico ou apresentam formas severas do HPTS têm indicação de tratamento cirúrgico, que consiste na paratireoidectomia
(PTX) (Custódio et al., 2013). Segundo as Diretrizes Brasileiras de Prática Clínica para o Distúrbio Mineral e Ósseo da DRC (DMO-DRC), as indicações de PTX são: pacientes com nível sérico de PTH persistentemente acima de 800 pg/mL, associado a uma ou mais condições clínicas como hipercalcemia e/ou hiperfosfatemia refratárias, calcificações extraósseas ou arteriolopatia urêmica calcificante (calcifilaxia) e doença óssea grave (Sampaio \& Moysés, 2011). Dados da literatura estimam que $5,5 \%$ a $30 \%$ dos pacientes necessitam do tratamento cirúrgico (Bashir et al., 2015; Oliveira et al., 2011). No Brasil, essa prevalência ainda é desconhecida. Um estudo brasileiro que avaliou 660 centros de diálise revelou que a prevalência de pacientes com PTH superior a 1.000 $\mathrm{pg} / \mathrm{mL}$ era de $10,7 \%$, valor esse subestimado se considerado o ponto de corte de $800 \mathrm{pg} / \mathrm{mL}$, sugerido pelas diretrizes brasileiras (Oliveira et al., 2011).

É importante destacar que a chegada de novas tecnologias como quelantes de P livres de Ca e alumínio, novos análogos do calcitriol e do cinacalcete mudou o tratamento do HPTS na última década. Especificamente em relação ao cinacalcete, esse é o único agente que atua no receptor de Ca das células paratireoides, reduzindo a liberação do PTH, sem os indesejáveis efeitos colaterais, como hipercalcemia e/ou hiperfosfatemia, frequentes com o uso de calcitriol e seus análogos (Custódio et al., 2013). O uso desses agentes aumentou substancialmente as chances de sucesso do tratamento clínico, reduzindo a necessidade da PTX (Messa et al., 2013).

Recentemente, o cinacalcete obteve um parecer favorável à incorporação pelo Sistema Único de Saúde (SUS) para o tratamento de pacientes com HPTS, com nível de PTH entre 800 e 1.500 pg/mL, após avaliação das evidências científicas disponíveis, assim como econômica e de impacto orçamentário [Ministério da Saúde (Brasil), 2015]. No entanto, para o 
cenário brasileiro, ainda não existem dados precisos que estimem a população elegível para o uso desse medicamento, bem como o custo da doença, dimensionando o impacto socioeconômico do tratamento clínico ou cirúrgico do HPTS nos pacientes não responsivos ao tratamento convencional (quelantes de $\mathrm{P}$ e calcitriol ou seus análogos).

Dessa forma, este estudo teve como objetivo estimar a população elegível para o tratamento de pacientes com HPTS, não controlados com a terapia convencional, bem como avaliar a utilização de recursos para o tratamento dessa população com cinacalcete ou PTX.

\section{Métodos}

Foi realizado um painel com 12 especialistas empregando a técnica Delphi, que estabelece os parâmetros-chave para o cálculo da população elegível para o uso de cinacalcete. Além disso, essa técnica foi utilizada para estimar as morbidades e recursos relacionados ao tratamento clínico e cirúrgico de pacientes em diálise com HPTS. Para tal, foram convidados nefrologistas especialistas no tratamento do DMO-DRC, além de cirurgiões de cabeça e pescoço especializados na realização de PTX, com o intuito de minimizar vieses de seleção.

Dentre os 12 especialistas recrutados para o painel, nove aceitaram participar da pesquisa. Do total, sete eram nefrologistas e dois eram cirurgiões de cabeça e pescoço, originários dos seguintes centros de referência: Hospital das Clínicas da Universidade de São Paulo, Hospital das Clínicas da Faculdade de Medicina de Ribeirão Preto, Hospital de Clínicas de Porto Alegre, Hospital do Rim - Fundação Oswaldo Ramos, Hospital Universitário Antônio Pedro, Hospital Universitário Pedro Ernesto da Universidade do Estado do Rio de Janeiro, Faculdade de Medicina da Universidade Federal de Uberlândia e Hospital das Clínicas da Universidade Federal de Pernambuco.

A pesquisa foi feita utilizando questionário estruturado enviado por meio eletrônico aos especialistas e encontro presencial para validação das informações coletadas; por fim, os resultados foram compilados de forma independente por pesquisador externo. O painel Delphi foi elaborado levando-se em conta as seguintes etapas de execução:

1. As informações referentes ao cálculo da população elegível e estimativa do uso de recursos foram enviadas, individualmente, a cada um dos especialistas participantes do painel, que responderam para o pesquisador - esse procedimento foi anônimo e confidencial;

2. As respostas foram escrutinadas e coletadas pelo pesquisador que compilou todas as respostas apresentadas em encontro presencial realizado dia 28 de agosto de 2014, em São Paulo, SP;

3. Nessa etapa, os especialistas foram solicitados a reconsiderar as informações e responder indicando sua concordância ou não com os itens apresentados;
4. As respostas foram coletadas mais uma vez e o processo foi repetido até o consenso ser alcançado. Adotou-se a técnica denominada de Delphi modificada, com um limite no número de ciclos de execução de até quatro ciclos para a busca do consenso (Spínola, 1981). Assim o "consenso" representou o nível obtido na última etapa determinada.

O questionário incluiu os dois objetivos principais do estudo: definição da população elegível (para cinacalcete e PTX) e uso de recursos.

Para definição da população elegível, os dados solicitados foram: população com DRC estádio 5, em diálise, com PTH superior a $800 \mathrm{pg} / \mathrm{mL}$, além de perguntas adicionais referentes ao percentual de pacientes em condições ideais para uso de cinacalcete e o percentual de pacientes atualmente encaminhados para PTX.

Para o uso de recursos, foram estimadas as seguintes condições: tratamento com cinacalcete (medicamentos e acompanhamento), PTX (pré-operatório, cirurgia e pós-operatório imediato e tardio), complicações da cirurgia (lesão do nervo laríngeo recorrente unilateral e bilateral, neuropraxia do laríngeo recorrente, pneumonia e sangramento), eventos cardiovasculares [infarto agudo do miocárdio (IAM) e acidente vascular encefálico (AVE)] e fratura de fêmur. Os especialistas foram orientados a estimar os percentuais de procedimentos ou medicamentos utilizados.

Foram considerados apenas os custos diretos relacionados aos cuidados prestados aos pacientes, baseados na tabela SUS. Os custos foram valorados em reais (R\$) para o ano de 2014.

Os custos relacionados ao período de hospitalização foram obtidos de informações do Sistema de Informações Hospitalares do SUS (SIH/SUS), processado pelo Datasus e gerido pelo Ministério da Saúde, utilizando como fonte primária de informação a Autorização de Internação Hospitalar (AlH) referente ao período de janeiro de 2013 a dezembro de 2013 [Ministério da Saúde (Brasil), s.d.]. Os custos unitários referentes à aquisição de medicamentos durante o período de acompanhamento ambulatorial foram obtidos do Banco de Preços em Saúde (BPS) [Ministério da Saúde (Brasil), 2013], utilizando-se o preço referente à média ponderada. Já para materiais, procedimentos e exames, avaliamos o Sistema de Gerenciamento da Tabela de Procedimentos, Medicamentos e Órteses, Próteses e Materiais Especiais do SUS (SIGTAP) [Ministério da Saúde (Brasil), 2014], com valores de referência para o ano de 2014.

Para a valoração de procedimentos como PTX, tratamento do IAM, AVE e fratura de fêmur, também foram considerados valores praticados pela tabela TUNEP (Tabela Única Nacional de Equivalência de Procedimentos - 2005), usada como referência para ressarcimento do SUS nos casos de atendimentos de beneficiários da rede pública de saúde e de planos de saúde privados. Tal procedimento teve como objetivo o desenvolvimento de uma análise de sensibilida- 
de, a fim de comparar os valores praticados pelo SUS versus aqueles dos planos privados.

\section{Resultados}

\section{Estimativa da população elegível ao uso de cinacalcete e PTX}

Na Tabela 1 estão descritos os cálculos para a obtenção da estimativa da população elegível ao tratamento com cinacalcete. Inicialmente foi calculada a porcentagem de pacientes com DRC, em diálise, com HPTS ( $n=40.480$ ), que correspondem a 44\% dos pacientes em diálise $(n=92.000)$, segundo o Censo Brasileiro de Diálise de 2011, realizado pela SBN (Custódio et al., 2013). Desses ( $n=40.480)$, os especialistas (Painel Delphi) estimaram que 25\% ( $n=10.120)$ têm níveis de PTH maiores que $800 \mathrm{pg} / \mathrm{mL}$, sendo, portanto, os pacientes com indicação ao tratamento com cinacalcete. Desse valor final, foram subtraídos $6 \%$ (percentual extraído com base no ensaio clínico EVOLVE) de pacientes que abandonam o tratamento devido a eventos adversos (EVOLVE Trial Investigators et al., 2012). Dessa forma, 9.513 pacientes configuram a população elegível ao tratamento com cinacalcete. Ainda de acordo com os especialistas, dos pacientes elegíveis ao tratamento medicamentoso do HPTS, 81\% ( $n=7.705)$ apresentavam condições ideais para uso do cinacalcete.

Com relação à PTX (Tabela 2), como esse era o único tratamento disponível no SUS para os pacientes com HPTS grave com níveis de PTH > $800 \mathrm{pg} / \mathrm{mL}$, estimou-se que 100\% dos pacientes eram indicados ao procedimento. Dessa forma, a

Tabela 1. Cálculo da população elegível e real com relação ao tratamento com cinacalcete

\begin{tabular}{|c|c|c|}
\hline $\begin{array}{l}\text { Número de pacientes } \\
\text { com DRC, em diálise }\end{array}$ & 92.000 & SBN, 2013 \\
\hline $\begin{array}{l}\text { \% de pacientes com DRC, } \\
\text { em diálise, com HPTS }\end{array}$ & $44 \%$ & SBN, 2013 \\
\hline $\begin{array}{l}\text { \% de pacientes com DRC, em diálise, } \\
\text { com HPTS e PTH > } 800 \text { pg/mL }\end{array}$ & $25 \%$ & Painel Delphi \\
\hline $\begin{array}{l}\text { \% de pacientes que abandonam } \\
\text { o tratamento com cinacalcete } \\
\text { devido a evento adverso }\end{array}$ & $6 \%$ & EVOLVE, 2012 \\
\hline $\begin{array}{l}\text { POPULAÇÃO ELEGÍVEL AO } \\
\text { TRATAMENTO COM CINACALCETE }\end{array}$ & 9.513 & \\
\hline $\begin{array}{l}\text { Pacientes com condições ideais } \\
\text { de uso do cinacalcete }\end{array}$ & $81 \%$ & Painel Delphi \\
\hline $\begin{array}{l}\text { POPULAÇÃO REAL INDICADA } \\
\text { AO USO DE CINACALCETE }\end{array}$ & 7.705 & \\
\hline
\end{tabular}

DRC: doença renal crônica; HPTS: hiperparatireoidismo secundário; PTH: paratormônio. ${ }^{1}$ EVOLVE Trial Investigators, et al. Effect of cinacalcet on cardiovascular disease in patients undergoing dialysis. N Engl J Med. 2012;367(26):2482-94. população elegível à PTX seria de 10.120 indivíduos. Desses, apenas $18 \%$ foram considerados com condições ideais para o tratamento cirúrgico $(n=1.822)$. No entanto, os especialistas consideraram que $76 \%(n=7.691)$ da população elegível à PTX é a população real encaminhada ao procedimento.

Na Tabela 3 estão descritas as condições apontadas pelos especialistas para o uso do cinacalcete em cenário ideal e real. Com relação ao cenário ideal (Tabela 3A), destacam-se o nível de Ca e/ou P adequados (apontado por 57\% dos especialistas), seguido pela contraindicação à PTX (43\%). Já em um cenário real (Tabela 3B), as condições apontadas foram aquelas referentes aos pacientes que têm Ca e/ou P fora de controle, ou controlados, mas que desenvolveram hipercalcemia e/ou hiperfosfatemia com uso do calcitriol (57\%), seguido pela presença de tumor marrom, fraturas, calcifilaxia e sintomas graves e incapacitantes, cada uma dessas apontadas por $29 \%$ dos especialistas, respectivamente.

Na Tabela 4 estão descritas as condições apontadas pelos especialistas para a indicação à PTX em cenário ideal e real. Com relação ao cenário ideal (Tabela 4A), 71\% dos especialistas apontaram a não resposta/intolerância ao tratamento medicamentoso, enquanto 29\% consideraram a ausência de eventos cardiovasculares e 14\% a exigência de centros especializados e capacitados. Já em um cenário real (Tabela 4B), as condições apontadas para indicação à PTX foram aquelas referentes aos pacientes com doença grave progressiva (calcifilaxia, tumor marrom) e calcificações de tecidos moles ou cardiovascular, destacadas por $100 \%$ dos especialistas. Hipercalcemia e/ou hiperfosfatemia refratárias ao tratamento

Tabela 2. Cálculo da população elegível, indicada e encaminhada à PTX

\begin{tabular}{|c|c|c|}
\hline $\begin{array}{l}\text { Número de pacientes } \\
\text { com DRC, em diálise }\end{array}$ & 92.000 & SBN, 2013 \\
\hline $\begin{array}{l}\text { \% de pacientes com DRC, } \\
\text { em diálise, com HPTS }\end{array}$ & $44 \%$ & SBN, 2013 \\
\hline $\begin{array}{l}\text { \% de pacientes com DRC, em diálise, } \\
\text { com HPTS e PTH > } 800 \text { pg/mL }\end{array}$ & $25 \%$ & Painel Delphi \\
\hline $\begin{array}{l}\text { \% de pacientes com DRC, em } \\
\text { diálise, com HPTS e PTH > } 800 \\
\text { pg/mL indicada à PTX }\end{array}$ & $100 \%$ & Painel Delphi \\
\hline POPULAÇÃO ELEGÍVEL PTX & 10.120 & \\
\hline $\begin{array}{l}\text { \% de pacientes com condições } \\
\text { ideais para realização da cirurgia }\end{array}$ & $18 \%$ & Painel Delphi \\
\hline POPULAÇÃO IDEAL INDICADA À PTX & 1.822 & \\
\hline $\begin{array}{l}\text { \% de pacientes atualmente } \\
\text { encaminhados à PTX }\end{array}$ & $76 \%$ & Painel Delphi \\
\hline $\begin{array}{l}\text { POPULAÇÃO REAL } \\
\text { ENCAMINHADA À PTX }\end{array}$ & 7.691 & \\
\hline
\end{tabular}

DRC: doença renal crônica; HPTS: hiperparatireoidismo secundário; PTH: paratormônio; PTX: paratireoidectomia. 
Tabela 3. Condições para uso de cinacalcete em um cenário ideal (A) ou real (B)

\begin{tabular}{|c|c|c|c|}
\hline A & & B & \\
\hline Condições & $\begin{array}{c}\% \text { de } \\
\text { especialistas* }\end{array}$ & Condições & $\begin{array}{c}\% \text { de } \\
\text { especialistas* }\end{array}$ \\
\hline Nível de cálcio e/ou fósforo adequados & $57 \%$ & $\begin{array}{l}\text { Pacientes que têm cálcio e/ou fósforo } \\
\text { fora de controle, ou controlados, mas } \\
\text { que desenvolvem hipercalcemia e/ou } \\
\text { hiperfosfatemia com o uso do calcitriol }\end{array}$ & $57 \%$ \\
\hline Contraindicação de paratireoidectomia & $43 \%$ & Tumor marrom & $29 \%$ \\
\hline Ausência de dor de forte intensidade e constante & $29 \%$ & Fraturas & $29 \%$ \\
\hline Ausência de fratura & $29 \%$ & Calcifilaxia & $29 \%$ \\
\hline Ausência de tumor marrom & $29 \%$ & Sintomas graves incapacitantes & $29 \%$ \\
\hline Pacientes aderentes ao tratamento e dieta & $14 \%$ & Progressão de calcificação ectópica & $14 \%$ \\
\hline $\begin{array}{l}\text { Pacientes que apresentem intolerância ao uso } \\
\text { dos ativadores do receptor da vitamina D }\end{array}$ & $14 \%$ & Progressão da perda da massa óssea & $14 \%$ \\
\hline- & - & Anemia resistente à eritropoietina & $14 \%$ \\
\hline
\end{tabular}

* Percentuais referentes a sete especialistas no total.

Tabela 4. Condições para indicação à PTX em um cenário ideal (A) ou real (B)

\begin{tabular}{|c|c|c|c|}
\hline A & & B & \\
\hline Condições & $\begin{array}{c}\% \text { de } \\
\text { especialistas* }\end{array}$ & Condições & $\begin{array}{c}\% \text { de } \\
\text { especialistas* }\end{array}$ \\
\hline $\begin{array}{l}\text { Não resposta/intolerância ao } \\
\text { tratamento medicamentoso }\end{array}$ & $71 \%$ & $\begin{array}{l}\text { Pacientes com doença grave progressiva } \\
\text { (calcifilaxia, tumor marrom) e calcificações } \\
\text { de tecidos moles e cardiovascular }\end{array}$ & $100 \%$ \\
\hline Ausência de eventos cardiovasculares & $29 \%$ & $\begin{array}{l}\text { Hipercalcemia (> 10,2 mg/dL) e/ou hiperfosfatemia } \\
\text { (> 6,0 mg/dL) refratárias ao tratamento clínico } \\
\text { e/ou eventos adversos causados por ele } \\
\text { (incluindo uso de cinacalcete e/ou calcitriol e/ } \\
\text { ou ativadores do receptor de vitamina D) }\end{array}$ & $57 \%$ \\
\hline Existência de centros especializados e capacitados & $14 \%$ & Sintomas graves & $43 \%$ \\
\hline - & - & Fraturas & $43 \%$ \\
\hline- & - & $\begin{array}{l}\text { Doença de alta remodelação óssea, osteíte fibrosa } \\
\text { ao Rx, progressão da perda de massa osteomuscular }\end{array}$ & $29 \%$ \\
\hline- & - & Anemia resistente à eritropoietina & $29 \%$ \\
\hline- & - & $\begin{array}{l}\text { Glândulas paratireoides aumentadas à US } \\
\text { (volume > } 500 \mathrm{~mm}^{3} \text { ou } 1 \mathrm{~cm} \text { de diâmetro) }\end{array}$ & $14 \%$ \\
\hline- & - & PTH intacto $>500 \mathrm{pg} / \mathrm{mL}$ & $14 \%$ \\
\hline- & - & Miocardiopatia dilatada-like & $14 \%$ \\
\hline
\end{tabular}

* Percentuais referentes a sete especialistas no total. Rx: raio x; US: ultrassonografia; PTH: paratormônio.

clínico e/ou eventos adversos causados por ele (incluindo uso de cinacalcete e/ou calcitriol e/ou ativadores do receptor de vitamina D) foram apontadas por $57 \%$ dos especialistas, seguidas por sintomas graves e fraturas (43\%).

\section{Utilização de recursos}

Na Tabela 5, encontram-se descritos os custos do tratamento com cinacalcete e do acompanhamento do paciente, incluindo consultas e exames laboratoriais. O custo anual total foi de R\$27.712,95. Para esse cenário, foi considerada a dose máxi- ma de cinacalcete indicada em bula (Mimpara, 2016). Além do preço do medicamento, considerou-se também o custo do acompanhamento, que incluiu apenas o custo da dosagem de Ca, pois, de acordo com os especialistas, os demais exames ou procedimentos, como consultas, estão contemplados na rotina de diálise à qual o paciente já é submetido.

Na Tabela 6, encontram-se o custo total da PTX, que foi de $R \$ 16.841,85$, sendo $R \$ 1.013,88$ do procedimento em si, somados os valores de $R \$ 496,73$ e $R \$ 15.331,74$, referentes ao período pré e pós-operatório, respectivamente. Com relação 
Tabela 5. Custo anual do tratamento com cinacalcete (medicamento e acompanhamento)

\begin{tabular}{lcccc}
\hline & \% de utilização & Quantidade & Custo unitário & Custo final \\
\hline Cinacalcete & $100 \%$ & $1890^{*}$ & $R \$ 14,66$ & $R \$ 27.707,40$ \\
\hline Consultas & $0 \%$ & 0 & $R \$ 10,00$ & $R \$ 0,00$ \\
\hline Cálcio (incremental) & $100 \%$ & 3 & $R \$ 1,85$ & $R \$ 5,55$ \\
\hline Fósforo & $0 \%$ & 0 & $R \$ 1,85$ & $R \$ 0,00$ \\
\hline PTH & $0 \%$ & 0 & $R \$ 2,01$ & $R \$ 0,00$ \\
\hline Fosfatase alcalina & $0 \%$ & 0 & $R \$ \mathbf{2 7 . 7 1 2 , 9 5}$ \\
\hline TOTAL & & & $R$
\end{tabular}

* Dose total anual de cinacalcete, considerando três semanas em média para cada ajuste de dose.

Tabela 6. Custo da paratireoidectomia

\begin{tabular}{lcccc}
\hline & \% de utilização & Quantidade & Custo unitário & Custo final \\
\hline Pré-operatório & $100 \%$ & 1 & $R \$ 496,73$ & $R \$ 496,73$ \\
\hline Cirurgia & $100 \%$ & 1 & $R \$ 1.013,38$ & $R \$ 1.013,38$ \\
\hline Pós-operatório & $100 \%$ & 1 & $R \$ 15.331,74$ & $R \$ 15.331,74$ \\
\hline TOTAL & & & & $R \$ 16.841,85$ \\
\hline
\end{tabular}

às complicações cirúrgicas, devido ao baixo percentual de ocorrência, o custo total ponderado foi de R\$ 17,14 (Tabela 7).

Para a fratura de fêmur (paciente não operado, com PTH acima de $800 \mathrm{pg} / \mathrm{mL}$ ), o custo ponderado final foi de R\$ 188,69, considerando um custo total de R\$1.257,90 aplicado a um percentual de ocorrência de 15\%. Para os eventos cardiovasculares, o custo ponderado para o tratamento do IAM e AVE foi de R\$149,72, considerando-se os percentuais de ocorrência com base no estudo EVOLVE (EVOLVE Trial Investigators et al., 2012). Eventos cardiovasculares como calcificações arteriais, valvares e coronarianas foram considerados não quantificáveis, ou seja, apenas "marcadores" de risco e morbidade da doença, portanto não foram considerados na análise de uso dos recursos.

\section{Análise de sensibilidade}

Uma análise de sensibilidade foi desenvolvida com o objetivo de comparar o custo do tratamento com cinacalcete em dois ce- nários principais: cenário de dose máxima (conforme estipulado em bula) versus o cenário que considerou a dose média do medicamento descrito no estudo EVOLVE (66,8 mg/dia). Os custos totais, para os diferentes cenários, foram de R\$27.712,95 e de R\$ $11.924,13$, respectivamente. Uma comparação entre os dois cenários demonstrou uma diferença de 57\%, com um incremento de $R \$ 1$ 15.788,82 para o cenário de dose máxima (Figura 1).

Uma comparação entre os custos dos procedimentos como PTX, tratamento do IAM, AVE e fratura de fêmur praticados pelo SUS e aqueles descritos na tabela TUNEP (2005) demonstrou grande defasagem entre os custos obtidos nessas duas fontes. Nesta análise, os custos individuais observados na tabela TUNEP, para cada procedimento, foram somados aos custos de diária de hospitalização (incluídos cuidados em terapia intensiva), gerando o valor total de cada procedimento. Em particular para fratura de fêmur, foram considerados 30 dias de tratamento conservador. Os custos finais calculados via TUNEP foram de R\$ 4.204,34 para PTX,

Tabela 7. Custo ponderado do manejo das complicações cirúrgicas

\begin{tabular}{|c|c|c|c|}
\hline & \% de ocorrência & Custo unitário & Custo final \\
\hline Lesão do nervo laríngeo recorrente unilateral & $1,00 \%$ & $\mathrm{R} \$ 122,54$ & $R \$ 1,23$ \\
\hline Lesão do nervo laríngeo recorrente bilateral & $0,50 \%$ & $\mathrm{R} \$ 187,94$ & $R \$ 0,94$ \\
\hline Hematoma & $1,36 \%$ & $R \$ 0,00$ & $R \$ 0,00$ \\
\hline Neuropraxia do laríngeo recorrente & $1,50 \%$ & $R \$ 122,54$ & $R \$ 1,84$ \\
\hline Infecção hospitalar (cirúrgico/PNM/ITU) & $4,00 \%$ & $\mathrm{R} \$ 171,93$ & $\mathrm{R} \$ 6,88$ \\
\hline Sangramento & $5,00 \%$ & $R \$ 125,28$ & $R \$ 6,26$ \\
\hline TOTAL & & & $\mathrm{R} \$ 17,14$ \\
\hline
\end{tabular}

PNM: pneumonia; ITU: infecção do trato urinário. 


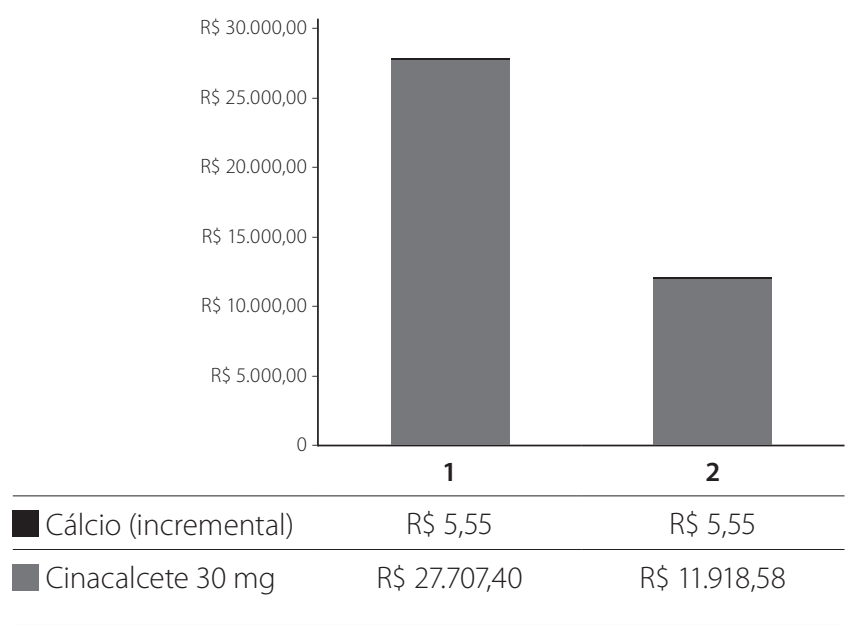

Figura 1. Comparação entre os custos totais anuais nos cenários de dose máxima (1) e dose utilizada de cinacalcete no estudo EVOLVE (2).

$\mathrm{R} \$$ 4.010,95 para IAM, $\mathrm{R} \$ 3.349,25$ para AVE e $\mathrm{R} \$ 12.796,50$ para fratura de fêmur (30 dias de tratamento). Comparados aos valores pagos pelo SUS, estes representaram um incremento de 77\%, 55\%, 63\% e 90\%, respectivamente.

\section{Discussão}

A Avaliação de Tecnologias em Saúde (ATS) compreende estudos e metodologias de avaliação sistemática das consequências da utilização de intervenções na saúde, tendo como principal propósito a qualificação dos processos para definição de prioridades, alocação de recursos e implantação de programas e políticas de saúde.

A Lei no 12.401/2011 introduziu mudanças importantes no processo de avaliação da incorporação de tecnologias em saúde no âmbito do SUS. Dentre elas, destaca-se a criação da Comissão Nacional de Incorporação de Tecnologias no SUS (Conitec) (Gadelha, 2003).

A Conitec, órgão colegiado de caráter permanente, integrante da estrutura regimental do Ministério da Saúde (MS), tem por objetivo assessorar o MS nas atribuições relativas a incorporação, exclusão ou alteração de tecnologias em saúde, bem como na constituição ou alteração de protocolos clínicos e diretrizes terapêuticas empregados no SUS.

Segundo a Conitec, os critérios usados para emissão de pareceres sobre incorporação de tecnologias no SUS incluem: a) busca ampla de estudos publicados e não publicados; b) avaliação crítica da melhor evidência disponível considerando desfechos clinicamente relevantes; c) necessidades médicas não atendidas da população e prioridades da política de saúde; d) caracterização do mercado e escolha de comparadores (alternativas terapêuticas); e) avaliação da logística e estrutura necessária para implantação da nova tecnologia; f) avaliação de estudo de custo-efetividade submetido pelo demandante; g) análise de impacto orçamentário.
Um exemplo da atuação da Conitec foi o processo de avaliação do medicamento cinacalcete para o tratamento do HPTS em pacientes com DRC, refratários ao tratamento convencional. No dia 5/9/2013, os membros da Conitec presentes na reunião do plenário deliberaram, por unanimidade, por não recomendar a incorporação ao SUS (Diário Oficial da União, 2013). Apesar da negativa, o plenário declarou que considerava necessária a revisão dos Protocolos Clínicos e Diretrizes Terapêuticas de Osteodistrofia Renal e Hiperfosfatemia do MS, momento em que o cinacalcete seria novamente avaliado quanto ao seu potencial benefício clínico para um grupo específico e bem delimitado de pacientes com DRC e HPTS.

O painel de especialistas descrito, composto por especialistas convidados e responsáveis pelos dados analisados neste artigo, objetivou responder à seguinte questão: "potencial benefício clínico para um grupo específico e bem delimitado de pacientes com DRC e HPTS", demandada pelos membros da Conitec, quanto ao grupo elegível para o uso do cinacalcete.

Os resultados descritos nesse artigo foram apresentados à Conitec, que decidiu pela recomendação favorável à incorporação do cloridrato de cinacalcete para pacientes com HPTS e DRC no âmbito do SUS, em setembro de 2015 (Diário Oficial da União, 2015).

A PTX ainda é o tratamento de escolha para pacientes com HPTS grave (Custódio et al., 2013) ou que se tornam refratários à terapia medicamentosa, apresentando resultados satisfatórios em termos de melhora da pressão elevada, anemia, status nutricional, metabolismo lipídico e glicídico, força muscular e da qualidade de vida (Goldenstein et al., 2013). Convém ressaltar que a PTX e o tratamento medicamentoso não são excludentes no cuidado do paciente com DRC e HPTS. Um número significativo de pacientes em fase precoce do HPTS será beneficiado com o uso de cinacalcete, com interrupção da progressão da doença e prevenção da necessidade de uma PTX e das complicações do HPTS avançado. Nos pacientes nessa fase da doença, o uso do cinacalcete pode representar uma terapia de suporte até a realização da PTX, e naqueles com HPTS avançado que não tenham condições clínicas para realizar a PTX, o uso do cinacalcete representa terapêutica útil, em relação ao tratamento convencional. (Komaba et al., 2012).

Destaca-se também que os resultados obtidos por meio do painel de especialistas indicam a lacuna terapêutica existente no SUS, até que ocorra a incorporação do cinacalcete para os pacientes com níveis de PTH acima de 800 pg/mL, uma vez que a realização da PTX é a única opção disponível. De acordo com os especialistas, estima-se que $76 \%$ dos pacientes nessas condições têm indicação para a realização da cirurgia. Contudo, apenas 18\% desses pacientes apresentam condições ideais para o procedimento. Dessa forma, como não há alternativas para o manejo dessa população, mais de 70\% dos pacientes são encaminhados para cirurgia no SUS. 
O HPTS é uma das mais graves complicações em pacientes com DRC, uma vez que está relacionado a eventos cardiovasculares, considerados a maior causa de mortalidade nessa população (Oliveira et al., 2011). Considerando-se que 44\% da população dialítica têm HPTS, o número de pacientes acometidos pela doença se eleva proporcionalmente ao número de pacientes em diálise (Custódio et al., 2013).

No Brasil, o HPTS ganhou atenção ao seu tratamento a partir da década de 1980. No entanto, o tratamento medicamentoso disponível no SUS (quelantes de P e calcitriol) não tem sido suficiente para controlar de maneira efetiva a doença. Dessa forma, a prevalência de casos graves de HPTS tem aumentado, principalmente porque a demanda de PTX não tem sido correspondida. Filas à espera da cirurgia podem ser observadas nos poucos serviços especializados e capacitados para a realização do procedimento. Além disso, a maior porcentagem de pacientes em espera na fila evolui para óbito, quando comparada àquela dos que são submetidos ao procedimento (Goldenstein et al., 2013). Essas filas, na sua maioria, são compostas por pacientes que não teriam indicação real ao procedimento cirúrgico caso o tratamento medicamentoso efetivo, com cinacalcete, estivesse disponível. De fato, é por esse motivo que, segundo o painel de especialistas desse estudo, se observa um número quase $70 \%$ maior de pacientes que são encaminhados à cirurgia, comparado ao que efetivamente teria indicação a ela. Essa cifra se aproxima da porcentagem de pacientes com real indicação ao uso de cinacalcete e que não têm acesso ao tratamento.

Esse cenário se agrava quando se observa que os pacientes com HPTS grave devem obrigatoriamente ser priorizados para a cirurgia, expondo assim um paciente grave (HPTS + doença cardiovascular) a um risco cirúrgico que poderia ser evitado. Se por um lado a PTX é um tratamento definitivo do HPTS, por outro devem-se considerar as complicações de médio e longo prazo que ocorrem após o procedimento; por exemplo, casos de recidiva do HPTS ou mesmo de hipoparatireoidismo com consequente agravamento da calcificação vascular são relatados na literatura (London et al., 2008). Por fim, a não prevenção do HPTS ou seu tratamento inadequado favorece a gravidade da doença, aumentando as comorbidades, piorando a qualidade de vida e comprometendo potencialmente $\mathrm{o}$ transplante renal futuro.

\section{Conclusão}

O resultado obtido por meio do painel com os especialistas permitiu calcular a população elegível e os custos totais do tratamento com cinacalcete e PTX. Além disso, estimou-se que $76,3 \%$ dos pacientes são indicados para a PTX por falta de outras opções terapêuticas para o tratamento do HPTS, ou seja, pacientes que são considerados não responsivos ao tratamento com as opções terapêuticas disponíveis no SUS.

\section{Referências bibliográficas}

Bashir SO, Omer HA, Aamer MA, Somialy R, Morsy MD. Tolerance and efficacy of a low dose of the calcimimetic agent cinacalcet in controlling moderate to severe secondary hyperparathyroidism in hemodialysis patients. Saudi J Kidney Dis Transpl. 2015;26(6):1135-41.

Cunningham J, Locatelli F, Rodriguez M. Secondary hyperparathyroidism: pathogenesis, disease progression, and therapeutic options. Clin J Am Soc Nephrol. 2011;6(4):913-21.

Custódio M. Comunicado do Comitê de Distúrbios Mineral e Ósseo da Doença Renal Crônica (DMO-DRC) da Sociedade Brasileira de Nefrologia (SBN). São Paulo: SBN; 2014

Custódio MR, Canziani MEF, Moysés RMA, Barreto FC, Neves CL, Oliveira RB, et al. Protocolo clínico e diretrizes terapêuticas para o tratamento do hiperparatireoidismo secundário em pacientes com doença renal crônica. J Bras Nefrol. 2013;35(4):308-22

Diário Oficial da União. Portaria no 50, de 15 de outubro de 2013. DOU no 201, de 16 de outubro de 2013, p. 65

Diário Oficial da União. Portaria n 48, de 29 de setembro de 2015. DOU n 187, de 30 de setembro de 2015, p. 71 .

EVOLVE Trial Investigators, Chertow GM, Block GA, Correa-Rotter R, Drüeke TB, Floege J, Goodman WG, et al. Effect of cinacalcet on cardiovascular disease in patients undergoing dialysis. N Engl J Med. 2012;367(26):2482-94.

Gadelha CAG. O complexo industrial da saúde e a necessidade de um enfoque dinâmico na economia da saúde. Ciênc Saúde Coletiva. 2003;8(2):521-35.

Goldenstein PT, Elias RM, Pires de Freitas do Carmo L, Coelho FO, Magalhães LP, Antunes $\mathrm{GL}$, et al. Parathyroidectomy improves survival in patients with severe hyperparathyroidism: a comparative study. PLoS One. 2013;8(8):e68870.

Komaba H, Moriwaki K, Goto S, Yamada S, Taniguchi M, Kakuta T, et al. Costeffectiveness of cinacalcet hydrochloride for hemodialysis patients with severe secondary hyperparathyroidism in Japan. Am J Kidney Dis. 2012;60(2):262-71.

Lima EM, Gesteira MFC, Bandeira MFS. Diretrizes do distúrbio do metabolismo mineral e ósseo na doença renal crônica da criança. J Bras Nefrol. 2011;33(2):189-247.

London GM, Marchais SJ, Guérin AP, Boutouyrie P, Métivier F, de Vernejoul MC. Association of bone activity, calcium load, aortic stiffness, and calcifications in ESRD. J Am Soc Nephrol. 2008;19(9):1827-35.

Messa P, Regalia A, Alfieri CM, Cresseri D, Forzenigo L, Gandolfo MT, et al. Current indications to parathyroidectomy in CKD patients before and after renal transplantation. J Nephrol. 2013;26(6):1025-32.

Ministério da Saúde (Brasil). Comissão Nacional de Incorporação de Tecnologias no SUS (Conitec). Cinacalcete e paricalcitol para o tratamento de pacientes com hiperparatireoidismo secundário à doença renal (HPTS), em diálise e refratários à terapia convencional - Relatório de recomendação. Brasília: Ministério da Saúde; 2015.

Ministério da Saúde (Brasil). Coordenação Geral de Economia da Saúde. Departamento de Economia da Saúde investimentos e desenvolvimento. Banco de Preços em Saúde. 2013.

Ministério da Saúde (Brasil). Datasus - Departamento de Informática do SUS. (s.d.). Windows - TabWin.

Ministério da Saúde (Brasil). Departamento de Informática do SUS (Datasus). SIGTAP - Sistema de Gerenciamento da Tabela de Procedimentos, Medicamentos e OPM do SUS. 2014.

Mimpara [bula]. São Paulo: Amgen Biotecnologia do Brasil Ltda.; 2016.

Oliveira RB, Silva EN, Charpinel DMF, Gueiros JEB, Neves CL, Sampaio EA, et al. Situação do hiperparatireoidismo secundário autônomo no Brasil: dados do censo brasileiro de paratireoidectomia. J Bras Nefrol. 2011;33(4):457-62.

Sampaio EA, Moysés RMA. Paratireoidectomia na DRC. J Bras Nefrol. 2011;33(2):189-247.

Spínola AWP. Delfos: proposta tecnológica alternativa São Paulo. Faculdade de Saúde Pública. Universidade de São Paulo. 1981. 